\title{
Tradução e adaptação semântica do Questionário de Controle Atencional para o Contexto Brasileiro
}

\author{
Translation and semantic adaptation of the \\ Attentional Control Scale for \\ the Brazilian Context
}

\author{
Alberto FILGUEIRAS \\ Bruno de Oliveira GALVÃO2 \\ Pedro PIRES ${ }^{3}$ \\ Ana Carolina Monneratt FIORAVANTI-BASTOS ${ }^{4}$ \\ Gabriela Pereira Rangel HORA ${ }^{5}$ \\ Cristina Maria Teixeira SANTANA ${ }^{6}$ \\ Jesus LANDEIRA-FERNANDEZ ${ }^{1}$
}

\begin{abstract}
Resumo
A capacidade de controlar a atenção é uma tarefa complexa que demanda diferentes processos mentais. O presente estudo teve como objetivo traduzir e adaptar o Questionário de Controle Atencional para o Brasil. A pesquisa foi realizada com a ajuda de dois painéis multidisciplinares: um formado por profissionais de Psicologia Experimental e Neurociências responsáveis por auxiliar nas adaptações semânticas, e outro de especialistas em Psicologia da Atenção responsáveis por julgar os itens em três aspectos: clareza, coerência e compatibilidade semântica. O Coeficiente de Validade de Conteúdo foi utilizado para avaliar os itens a partir dos escores desses juízes. Dentre os 20 itens, apenas três não se mantiveram acima do critério de 0,80: itens 3, 16 e 20. Os resultados foram discutidos considerando os problemas enfrentados na adaptação do instrumento. Concluiu-se que o Questionário de Controle Atencional foi traduzido adequadamente e apresenta equivalência semântica com seu original estadunidense.
\end{abstract}

Palavras-chave: Adaptação; Aplicação do teste; Atenção.

$\boldsymbol{\nabla} \boldsymbol{\nabla} \boldsymbol{\nabla} \boldsymbol{v}$

1 Pontifícia Universidade Católica do Rio de Janeiro, Departamento de Psicologia, Programa de Pós-Graduação em Psicologia Clínica. R. Marquês de São Vicente, 225, Edifício Cardeal Leme, Sala 201, Gávea, 22453-900, Rio de Janeiro, RJ, Brasil. Correspondência para/Correspondence to: J. LANDEIRA-FERNANDEZ. E-mail: <landeira@puc-rio.br>.

2 Centro Universitário Augusto Motta, Departamento de Psicologia. Rio de Janeiro, RJ, Brasil.

${ }^{3}$ Centro Universitário Celso Lisboa, Departamento de Psicologia. Rio de Janeiro, RJ, Brasil.

${ }^{4}$ Universidade Federal Fluminense, Departamento de Psicologia. Rio de Janeiro, RJ, Brasil.

${ }^{5}$ Universidade Federal do Rio de Janeiro, Programa de Pós-Graduação em Psicologia. Rio de Janeiro, RJ, Brasil.

${ }^{6}$ Pontifícia Universidade Católica do Rio de Janeiro, Departamento de Psicologia, Programa de Pós-Graduação em Psicologia Clínica com Ênfase em Psicometria. Rio de Janeiro, RJ, Brasil. 


\begin{abstract}
The ability to control attention is a complex task that requires different mental processes. The present study aims to translate and adapt the Attentional Control Scale to Brazil. The research was conducted with the support of two multidisciplinary panels: one formed by Experimental Psychologists and Neuroscientists who were responsible to help throughout the semantic adaptation process, and another one formed by Attention Psychology specialists responsible to judge items in three aspects: comprehensiveness, coherence and semantic compatibility. The Content Validity Coefficient was used to assess items from judges scores. Among 20 items, only three did not hold values above the 0.80 criteria: items 3, 16 and 20. Results were discussed considering the problems faced throughout the adaptation of the instrument. It was concluded that the Attentional Control Scale was adequately translated and it shows semantic equivalency with the American original scale.
\end{abstract}

Keywords: Adaptation; Testing; Attention.

Em meio à multidão, podem-se ouvir diversas vozes e sons de diferentes naturezas. Porém, quando se está conversando com alguém, é possível manter-se atento concentrando-se somente no que está sendo dito e suprimir os demais estímulos sonoros. O mesmo acontece com todos os outros inputs sensoriais. Ao se provar um prato com muitos sabores, privilegia-se o sabor mais agradável ao paladar em relação aos demais. Ao olhar para uma figura com grande quantidade de informações visuais, focalizam-se os aspectos que mais exigem dos sentidos. Durante um concerto de rock, ao experimentar vários estímulos hápticos, é possível diferenciar aqueles que não são relevantes daqueles que estão associados a uma intenção, como, por exemplo, um toque no ombro para chamar alguém. Em uma perfumaria, consegue-se distinguir as fragrâncias optando por concentrar naquelas que mais agradam. Todas essas sensações possuem inúmeras variáveis que influenciam na concentração em um determinado estímulo, escolhendo-o no meio de tantos outros que competem para serem percebidos. A esse filtro, ou mecanismo de focalizar em um estímulo ignorando os demais, dá-se o nome de Atenção.

Historicamente, a atenção propriamente dita começou a ser estudada pelo psicólogo Edward Colin Cherry em 1953. À época, esse pesquisador buscou compreender o fenômeno da escuta seletiva no paradigma do experimento da festa do coquetel - the cocktail party problem. As primeiras descobertas sugeriram que variáveis físicas, como volume e proximidade, podem influenciar a capacidade de focalizar um diálogo no meio de vários outros. Porém, outras características, como interesse no assunto ou a história pessoal do ouvinte, podem gerar uma escuta seletiva, mesmo que seja direcionada a conversas que estão mais longe ou que se dão em volume mais baixo (Gazzaniga \& Heatherton, 2007).

A atenção visual, por sua vez, foi utilizada pela primeira vez como palavra-chave em 1976. A partir daí, o crescimento da atenção visual enquanto campo de pesquisa deu-se exponencialmente ao longo das últimas quatro décadas. Desde 1980, foi atingida a impressionante marca de 2400 artigos utilizando 'atenção visual' como palavra-chave, apenas na base de dados da PubMed (Carrasco, 2011). Esse tipo de atenção passou a constituir a maior parte do repertório de conhecimento sobre os mecanismos atencionais.

De acordo com Derryberry (2002), o controle da atividade atencional é mediado pelo autocontrole voluntário. Estaria, portanto, relacionado mais especificamente a elementos da atenção executiva dentro do modelo de Posner e Rothbart (1998). O modelo sugere que há atividade pré-perceptiva, chamada de sistema atencional anterior, relacionada à trajetória das respostas e dos comportamentos automáticos. Esse sistema assemelha-se ao conceito de priming e é influenciado pelos mecanismos pré-atencionais nos processamentos superiores (Kiefer, Adams, \& Zovko, 2012). Na definição de Kolb e Wishaw (2008) o priming é um fenômeno associado à memória implícita - captada em processos automáticos da atenção -, no qual a exposição prévia a um estímulo influencia a resposta a um estímulo posterior. Um exemplo de priming ocorre quando 
uma pessoa, solicitada a falar palavras que começam com a letra " $A$ ", provavelmente será influenciada pelos objetos que estão presentes no mesmo ambiente: abajur, almofada etc.

Contudo, esse paradigma teórico sugere que o priming não é o mecanismo responsável nos processos subjacentes à consciência, mas faz parte, junto a outros mecanismos, do sistema atencional anterior que, por sua vez, coordena as atividades pré-perceptivas (Derryberry, 2002). Segundo esse ponto de vista, os sistemas de vigilância e atenção voluntária seriam posteriores e influenciados pelo sistema atencional anterior. Este percorre estruturas mais primais no cérebro e relaciona-se mais fortemente aos sistemas motivacionais rudimentares, como ansiedade e medo (Derryberry, 2002; Posner \& Rothbart, 1998). Como sugerem Posner e Rothbart (1998), a atenção voltada para a execução de tarefas teria componentes tanto automáticos quanto voluntários, e estaria sujeita a alterações ao longo da janela temporal executiva, em virtude das oscilações emocionais do sujeito.

A atenção apresenta dois diferentes circuitos para o seu funcionamento. O sistema automático ou sistema atencional anterior, que gera a orientação da atenção primária e percorre um caminho de estruturas filogeneticamente mais antigas, como o córtex cingulado, responsável pelo sistema de atenção automática (Posner \& Rothbart, 1998), e a amígdala, responsável pelo sistema de vigilância e prontidão (Irwin et al., 1996). Esse circuito é voltado para a orientação rápida e imediata a um estímulo que demanda uma resposta automática, como uma ameaça de ataque ou risco iminente. A atividade desse sistema é considerada bottom up, ou ascendente, por não acessar a consciência ou a atividade cognitiva superior antes da resposta (Derryberry, 2002). Já o processo top down ou descendente, embora também utilize os recursos do sistema atencional anterior, possui como principal referência a via aferente do córtex cingulado posterior para o córtex pré-frontal medial (Small et al., 2003). De acordo com esse processo, a atenção voluntária gera uma tomada de decisão à medida que acessa a consciência, funcionando como um sistema supervisor de retroalimentação entre a inibição e/ou a execução da resposta correta. Após a execução ou inibição da resposta, uma nova via se encaminha para a tomada de consciência, e um processo de retroalimentação é ativado para julgar se aquela foi a melhor solução diante da demanda do estímulo (Norman \& Shallice, 1986). A Figura 1 representa os dois sistemas em face dessa perspectiva teórica.

De acordo com Posner (1980), a capacidade de alternar a atenção é um processo de duas vias automática e voluntária. O processo automático é um processo ascendente usado para orientar a atenção a partir de dicas ambientais e para manter o foco dependendo de sua relevância. Em tarefas de orientação visuoespacial, Posner demonstrou como a atenção pode ser automatizada quando associada a uma pista visual (Posner, 1980). Essas evidências foram confirmadas em diversos paradigmas experimentais e independem do tipo de resposta motora (Conde, Filgueiras, \& Lameira, 2009). Os processos de alternância do foco atencional variam de acordo com o tipo de pista, podendo ser sustentado ao longo do tempo (Filgueiras, 2010) e influenciado pelas características físicas dos estímulos (Filgueiras, 2011).

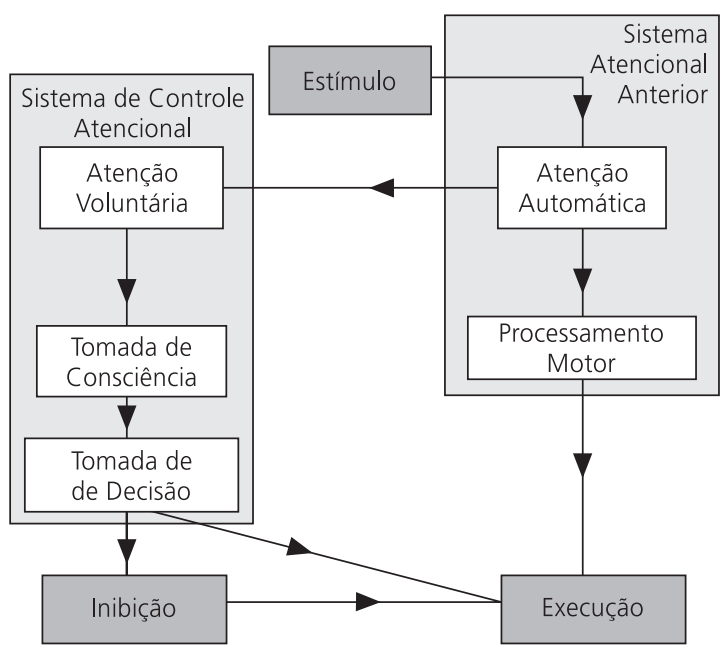

Figura 1. Esquema cognitivo na teoria do sistema atencional supervisor.

Nota: Esquematização da trajetória do estímulo até a execução ou inibição da resposta. Diferença dos caminhos do Sistema Atencional Anterior e do Sistema de Controle Atencional, com base nos estudos de Derryberry (2002), Norman e Shallice (1986) e Posner e Rothbart (1998). 
A atenção voluntária, por outro lado, é aquela usada para monitorar e conduzir escolhas atencionais quando algo precisa ser privilegiado em detrimento daquilo a que naturalmente - automaticamente -, dar-se-ia mais atenção. Por exemplo, nos paradigmas de Posner (1980), corroborados pela literatura (Conde et al., 2009; Filgueiras, 2010; Filgueiras, 2011), quando se mudam as pistas/dicas ambientais para direções incongruentes ou incompatíveis com a resposta correta, as pessoas normalmente não erram, mas levam mais tempo para responder. De acordo com os teóricos, essa diferença no tempo de resposta se dá por uma reestruturação cognitiva descendente, que leva em conta as regras de responder para determinados estímulos independentemente das pistas ambientais. Nesse caso, trata-se de um processo descendente e voluntário - a pessoa quer responder corretamente -, que prepondera sobre o processo automático, mesmo que leve mais tempo para dar uma resposta satisfatória.

Todas essas pesquisas, no entanto, lançam mão de paradigmas experimentais orientados a responder perguntas ou hipóteses bastante específicas. Além disso, parecem ser medidas mais associadas ao desempenho dos participantes nas tarefas propostas, deixando em segundo plano o possível impacto no cotidiano desses sujeitos (Conde et al., 2009; Filgueiras, 2011; Posner, 1980). Em última instância, são pesquisas que levantam questões quanto aos processos psicológicos básicos, não exatamente se preocupando com sua importância clínica ou aplicada.

Na contramão dessa lógica, há pesquisas que buscam investigar as inter-relações dos processos atencionais com os fenômenos cotidianos. Filgueiras (2010) usou um paradigma experimental de atenção - medida dependente do desempenho do sujeito na tarefa -, para medir o impacto de um protocolo de treinamento mental em atletas de futebol de campo. Na Psicologia Clínica, Efstratopoulou, Janssen, e Simons (2012) desenvolveram uma medida - a Motor Behavior Checklist -, na forma de escala Likert, na qual pais, professores e cuidadores respondiam sobre o comportamento da criança a

176 fim de identificar a presença de risco de Transtorno de Déficit de Atenção e Hiperatividade (TDAH). Isso significa que muitos usos, além de pesquisas básicas, podem ser dados a instrumentos de medida que possuem a atenção ou o processo atencional como construto. O campo da avaliação psicológica dentro do contexto da Psicologia do Esporte (Filgueiras, 2010) e da Psicologia Clínica (Efstratopoulou et al., 2012) pode ser beneficiado por medidas que estejam mais vinculadas aos impactos dos processos atencionais no cotidiano das pessoas.

\section{Medidas da atenção}

Nas pesquisas da área da Atenção - em especial a orientação automática e voluntária da atenção visual -, é comum ver protocolos computadorizados sendo usados como método de mensuração. Os pesquisadores Posner, Snyder e Davidson (1980) propuseram um método de coleta de dados por tempo de reação motora manual, no qual os participantes respondiam a estímulos espacialmente distribuídos em uma tela de computador. Os estudos iniciais de Posner et al. (1980) - um método de coleta de dados comportamentais -, foi criado para diferentes condições experimentais da atenção visual e, mais tarde, da atenção seletiva auditiva (Kimchi, 1982). Esse método tornou-se consagrado na literatura e culminou em dois instrumentos validados e padronizados para amostras brasileiras, mais especificamente: o Teste de Computadorizado de Atenção Visual (TAVIS) (Duchesne \& Mattos, 1997) e o Teste Computadorizado de Atenção (TCA Visual) (Schmidt \& Manhães, 2001).

Contudo, há outros métodos para mensurar a atenção que não por paradigmas computadorizados. Baterias de inteligência e funções mentais também trazem testes de atenção ou resistência à distração, como a Bateria de Funções Mentais para Motorista (BFM) (Tonglet, 1999), a Bateria Geral de Funções Mentais (BGFM) (Tonglet, 2003) e as escalas Wechsler de inteligência para adultos e crianças, respectivamente Wechsler Adult Intelligence Scale III (Nascimento, 2004) e Wechsler Intelligence Scale for Children III (Figueiredo, Pinheiro, \& Nascimento, 1998). 
Para medir diferentes dimensões atencionais, os instrumentos validados e padronizados para amostras brasileiras e aprovados para uso pelo Conselho Federal de Psicologia (Conselho Federal de Psicologia, 2012) são: o teste Atenção Concentrada (AC) (Cambraia, 2003) o Teste de Atenção Dividida (AD) e Sustentada (AS) (Sisto, Noronha, Bartholomeu, \& Rueda, 2006), o Teste D2 (Brickenkamp, 2000), a Escala de Atenção Seletiva Visual (EASV) (Sisto \& Castro, 2010), o Teste de Atenção Seletiva (TAS) (Camilo, 2012), o Teste de Atenção Concentrada (TEACO-FF) (Rueda, 2008), o Teste de Atenção Dividida (TEADI) (Rueda, 2009a) e o Teste de Atenção Alternada (TEALT) (Rueda, 2009b). A característica comum a esses testes é que todos solicitam ao sujeito executar uma tarefa determinada previamente, atrelando seu nível de atenção a seu desempenho.

Há, no entanto, escalas tradicionais que usam o preenchimento de terceiros ou escalas de autopreenchimento baseadas em comportamentos observados ao longo do cotidiano do sujeito. A já citada Motor Behavior Checklist (Efstratopoulou et al., 2012) normalmente é usada para medir níveis de atenção em crianças a fim de diagnosticar TDAH. O preenchimento é feito por terceiros e não pela criança, de modo que não constitui autopreenchimento.

Todavia, algumas escalas tentam quantificar a atenção usando a coleta por autopreenchimento. Por exemplo, o Cognitive Failures Questionnaire (CFQ) (Broadbent, Cooper, FitzGerald, \& Parkes, 1982) é um instrumento de 25 itens que verifica, através de uma escala de autopreenchimento, problemas relacionados a duas valências cognitivas: memória e atenção. A Effortful Control Scale (ECS) (Lonigan \& Phillips, 2001) é uma escala de 24 itens que avalia paralelamente a capacidade de resistir à distração e à impulsividade. Essas medidas são evidências de que é possível avaliar a Atenção sem necessariamente depender de mensurar a aptidão direta do sujeito em uma tarefa, o que exige condições ambientais controladas.

Por outro lado, as escalas de autopreenchimento - nas quais o sujeito responde a perguntas com respostas fechadas, normalmente do tipo Likert, sobre si mesmo -, existem em maior número que as escalas de preenchimento por terceiro, nas quais uma terceira pessoa responde a perguntas fechadas sobre o comportamento do sujeito. No entanto, as escalas de autopreenchimento existem em bem menor número que os paradigmas computadorizados e os testes em papel, os quais contemplam condições padronizadas para avaliar a aptidão ou a habilidade do sujeito em uma tarefa específica. Conforme a literatura, esse fenômeno provavelmente ocorra porque as escalas de autopreenchimento possuem menor precisão que os testes padronizados (Cronbach, 1971). No setting clínico, um psicólogo até pode diminuir a quantidade de distratores em seu consultório - retirando quadros e pintando a parede de cor neutra. No entanto, em outros ambientes, tais como a sala de espera, uma sala de ludoterapia ou um escritório dividido com outros profissionais, é impossível garantir que elementos capazes de distrair o sujeito sejam totalmente suprimidos, o que pode influenciar o resultado obtido em testes de desempenho (Carrasco, 2011). As escalas de preenchimento fornecem instrumental ao profissional de Psicologia para atuar nos mais diversos ambientes, sem a demanda de controlar integralmente o local de aplicação.

\section{Attentional Control Scale}

O presente estudo propõe uma escala de autopreenchimento como alternativa às medidas que usam o desempenho do sujeito para mensurar sua atenção. A Attentional Control Scale (ACS) (Derryberry \& Reed, 2002) é uma escala de autopreenchimento muito utilizada na literatura para mensurar a capacidade de controlar o foco atencional e mantê-lo ao longo de um período significativo de tempo. Possui 20 itens a serem respondidos numa escala do tipo Likert de quatro categorias: (1) quase nunca, (2) às vezes, (3) frequentemente e (4) sempre. São exemplos de itens da ACS em português brasileiro: "Tenho dificuldade de me concentrar em uma tarefa difícil quando há muito barulho em volta", "Quando estou concentrado, ignoro as sensações de fome e sede", ou "Para mim, é fácil ler ou escrever ao mesmo tempo em que falo ao telefone". 
A escala tem sido utilizada para estudos da manutenção da atenção com diferentes níveis de ansiedade. Em conformidade com Derryberry e Reed (2002), a manutenção da concentração é um aspecto relevante para a boa execução de uma tarefa. Quando uma pessoa apresenta níveis elevados de ansiedade, a ativação do sistema nervoso para lidar com a emoção faz com que haja um rebaixamento da capacidade de manter o foco em uma tarefa. Esse processo resulta na queda da atenção. O trabalho de Derryberry e Reed (2002) mostra que os valores da ACS tendem ser menores em pessoas que apresentam mais comportamentos ansiosos e maiores índices de estados de ansiedade. A correlação de Pearson na amostra entre a ACS e a medida de ansiedade foi de $r=-0,42$, sugerindo que a correlação entre o controle atencional e o estado de ansiedade é moderadamente inversa.

Esse estudo inaugurou os achados acerca das propriedades da ACS como um instrumento de medida da capacidade de o sujeito manter o controle da atenção ao longo de tarefas que demandam diferentes estados emocionais. Nesse contexto, a ACS surgiu a partir da necessidade de uma escala capaz de mensurar o controle atencional tanto no aspecto automático quanto no voluntário, examinando as relações entre atenção e emocionalidade (Derryberry, 2002; Derryberry \& Reed, 2002).

Nesse espectro de estudos, Spada, Georgiou e Wells (2010) utilizaram a ACS para examinar as relações entre controle atencional, ansiedade e metacognição. Os pesquisadores encontram evidências de que pensamentos negativos sobre falta de controle pessoal e perigo podem causar impacto no desempenho do controle atencional e gerar aumento nos índices dos estados de ansiedade.

Ólafsson et al. (2011) conduziram um trabalho semelhante ao de Derryberry e Reed (2002), ao comparar sintomas de depressão e ansiedade com o controle atencional medido a partir da ACS. Nesse estudo, foi usada a versão traduzida e adaptada do instrumento para o islandês, constituindo o primeiro estudo de adaptação transcultural da escala. Os resultados apontaram, assim como em Derryberry e Reed (2002), correlações inversamente 178 moderadas entre sintomas ansiosos e a ACS da ordem de $r=-0,45$, enquanto para a depressão tal relação é um pouco menor, $r=-0,37$.

Os autores promoveram ainda o estudo dos fatores da escala através de duas técnicas estatísticas: Análise Fatorial Exploratória (AFE), conduzida pelo método de Análise de Componentes Principais $(A C P)$; e Análise Fatorial Confirmatória (AFC), utilizando a técnica de estimação de Máxima Verossimilhança (MV). Os modelos foram ajustados a partir da matriz de covariâncias, permitindo que os erros de alguns itens fossem correlacionados. Os resultados tanto da AFE quanto de AFC apontaram melhor ajuste do modelo de 2 fatores, correlacionando o item 3 com os itens 6 , 7 e 8, o item 4 com o item 5, e o item 17 com o item 18. Os fatores encontrados na ACS foram: (1) foco, consistente na capacidade de a pessoa manter-se atenta ao longo de uma janela temporal específica, e (2) alternância, consistente na capacidade de a pessoa alternar o foco atencional para diferentes estímulos diante das demandas do ambiente (Ólafsson et al., 2011).

Os dados obtidos em estudos com a ACS (Derryberry \& Reed, 2002; Ólafsson et al., 2011) convergem com as evidências sobre atenção automática e voluntária de Posner (1980) e Posner e Rothbart (1998), assim como com as teorias de Derryberry (2002) e Norman e Shallice (1986) de que o processamento da atenção é em parte automático e em parte voluntário. Isso exige um sistema de supervisão para alternar o foco atentivo ou inibir sua alternância, enquanto focaliza por mais tempo somente um elemento ou um grupo específico de estímulos. Ao se basear nos resultados de Ólafsson et al. (2011), acredita-se que a ACS é capaz de abarcar as duas dimensões da atenção - automática e voluntária.

A Attentional Control Scale, ao avaliar os dois fatores da atenção, torna-se uma medida relevante no contexto da avaliação psicológica. Por meio de estudos com essa escala, psicólogos podem utilizá-la em diversos contextos sem precisar necessariamente do controle do ambiente. Ademais, os resultados de cada sujeito na ACS são capazes de oscilar de acordo com a ansiedade (Derryberry \& Reed, 2002; Ólafsson et al., 2011), proporcionando 
uma medida indireta na detecção de estímulos ansiogênicos no setting clínico. Diante desse prisma de possibilidades que essa escala pode proporcionar à Psicologia, o objetivo do presente estudo foi traduzir e adaptar semanticamente a ACS para o contexto do português brasileiro dentro dos modelos teóricos de Derryberry (2002) e Norman e Shallice (1986).

\section{Método}

\section{Participantes}

Participaram do estudo quatro especialistas em Psicologia Experimental e Neurociências, membros da equipe multidisciplinar para conduzir o processo de adaptação cultural. Ainda participaram quatro outros especialistas em Psicologia Experimental com notório conhecimento do sistema de controle atencional para o julgamento dos itens.

\section{Instrumentos}

Foi utilizada a versão original em inglês da ACS, com autorização dos autores. Duas versões traduzidas do inglês para o português foram usadas para dar forma à primeira adaptação da medida para o português brasileiro. A retrotradução para o inglês da versão islandesa da ACS foi usada como referência durante o processo de decisão das versões traduzidas. Finalmente, uma retrotradução do português para o inglês foi utilizada para ser avaliada e dar forma final à versão brasileira da escala.

\section{Procedimentos}

O primeiro passo foi solicitar a autorização do uso do Questionário para os autores, obtendo a resposta e o encaminhamento do instrumento através da professora Marjoire Reed, da Universidade do Estado do Óregon, Estados Unidos, por meio digital, no dia 6 de abril de 2012. Buscou-se ainda a versão retrotraduzida para o inglês do instrumento em islandês, gentilmente cedida pelo professor
Ragnar P. Ólafsson, da Universidade da Islândia, para eventuais esclarecimentos de dúvidas durante o processo de adaptação.

A tradução e adaptação cultural da ACS seguiu o planejamento sugerido por Flaherty et al. (1988). Dois tradutores brasileiros, oficialmente proficientes em inglês, conduziram a tradução do inglês para o português brasileiro. Uma comissão formada por quatro especialistas em Psicologia Experimental e Neurociências, todos proficientes em inglês e português, escolheu entre as traduções confeccionadas pelo primeiro ou segundo tradutor, permanecendo a questão em português que mais se aproximava semanticamente do conteúdo da versão original. Em casos de indecisão quanto à versão em português de um item, a sugestão dos especialistas foi considerada para confeccionar a versão final.

A partir da versão final da tradução para o português brasileiro, a retrotradução foi realizada por um tradutor norte-americano nativo, proficiente em português. Ambas as versões, somadas à versão retrotraduzida islandesa, foram comparadas pelo painel multidisciplinar e julgadas para confirmar a equivalência semântica da versão original em inglês, com a versão retrotraduzida islandesa e com a versão retrotraduzida brasileira.

A fim de examinar a clareza da escala, a coerência de cada item para avaliar o construto proposto assim como a qualidade da retrotradução, foi utilizado o Coeficiente de Validade de Conteúdo (CVC), como sugerido por Hernández-Nieto (2002). Quatro especialistas em Psicologia Experimental, Psiquiatria e Neuropsicologia, proficientes em inglês foram convidados pelo painel multidisciplinar para atribuir valores numa escala Likert de cinco categorias, de 0 (não concordo) a 4 (concordo plenamente). Os especialistas responderam aos seguintes itens referentes a clareza, coerência e retrotradução, respectivamente: (1) "Este item é de fácil compreensão", (2) "Este item está medindo uma dimensão do Controle Atencional", (3) "A versão original e a retrotradução têm valor semântico equivalente". Ao final do julgamento, foi utilizado o critério de $C V C \geq 0,80$, estabelecido por Hernández-Nieto (2002), para considerar se os itens foram ajustados semanticamente. 
O Coeficiente de Validade de Conteúdo é um índice proposto por Hernández-Nieto (2002) para quantificar e interpretar o julgamento de itens e escalas por um grupo de especialistas no construto que o instrumento propõe medir. Um CVC foi calculado para cada um dos aspectos a serem julgados na adaptação da escala: clareza, coerência com o construto e correspondência semântica da retrotradução. Ainda foi calculado um CVC para cada item da escala. Os valores de CVC aceitos por Hernández-Nieto (2002) para considerar a qualidade de um aspecto ou um item julgado devem ser maiores que 0,80 .

O coeficiente para cada item $\left(C V C_{i}\right)$ é calculado através da divisão da média dos valores dos julgamentos dos juízes $\left(\Sigma_{j}^{\times}\right)$pelo valor máximo da última categoria da escala Likert $\left(\mathrm{V}_{\max }\right)$ para um determinado item $x$. O CVC total da escala (CVC $)$ é dado pela subtração do CVC dos juízes (CVC) para a escala como um todo pelo Erro Padrão (Pe) da polarização dos juízes. O CVC é a divisão da média total dos escores (atribuídos a todos os itens da escala) pelo valor máximo da escala Likert. $\mathrm{Pe}_{\mathrm{j}^{\prime}}$ por sua vez, é calculado pela razão entre 1 e o número absoluto de juízes $\left(\mathrm{N}_{\mathrm{i}}\right)$, elevada ao próprio número absoluto de juízes. A Figura 2 apresenta as equações para o cálculo dos índices: $C V C_{i}, C V C_{j}$, $\mathrm{Pe}_{\mathrm{j}}$ e $C V \mathrm{C}_{t}$ e o algoritmo utilizado no presente estudo. As análises foram conduzidas no software Microsoft Office Excel.

Todos os procedimentos do projeto foram encaminhados para o Comitê de Ética do Departamento de Psicologia da Pontifícia Universidade Católica do Rio de Janeiro, obtendo parecer favorável sob o número 010/2012. O presente estudo somente se iniciou depois receber a aprovação do Comitê acima referido.

\section{Resultados}

A versão final da ACS para o português do Brasil recebeu o título de Questionário de Controle Atencional (QCA). O QCA apresenta os mesmos 20 itens da escala original, organizada na mesma ordem. Na adaptação semântica para a cultura brasileira, apenas quatro itens (20\%) necessitaram de
(1) Cálculo de $C V C_{i}$ para cada item da escala:

$$
C V C_{i}=\frac{\Sigma_{j}^{x}}{V_{\max _{x}}}
$$

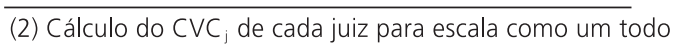

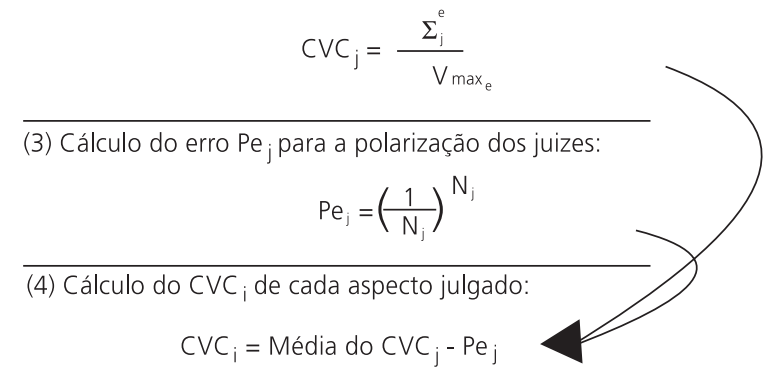

Figura 2. Algoritmo de cálculo do CVC.

Nota: Apresentação do algoritmo utilizado no presente estudo para calcular o valor do CVC para cada item (CVC) e do CVC total (CVC) para os aspectos: clareza global da escala, coerência com o construto e correspondência semântica da retrotradução.

CVC: Coeficiente de Validade de Conteúdo.

ajuste. Os itens 4, 5 e 6 trazem no seu conteúdo original a palavra "room". O painel multidisciplinar estabeleceu a expressão "ambiente fechado" em vez de "quarto" ou "cômodo". O item 12 na escala original apresenta a palavra "lecture", que pode significar tanto uma palestra ou conferência quanto uma aula. Nesse sentido, a equipe de especialistas sugeriu que a expressão fosse substituída por "palestra ou aula" na versão em português do Brasil, proporcionando ao respondente a clareza necessária para responder ao item. Os demais itens foram escolhidos pelo painel multidisciplinar entre as versões traduzidas e foram mantidos como sugerido pelos tradutores, com ajustes mínimos.

O Coeficiente de Validade de Conteúdo para toda a escala, considerando a média do $\mathrm{CV}_{\mathrm{t}}$ para cada um dos três aspectos julgados - clareza, coerência e retrotradução -, foi 0,85. Para a clareza dos itens, o $C V C_{t}$ foi 0,87. Para a coerência entre o conjunto de itens da escala como um todo e o construto que ela propõe avaliar - controle atencional -, o valor do $C V C_{t}$ foi 0,83 . Finalmente, para a equivalência semântica entre a versão original e a retrotradução, o CVC foi $_{t}$ 0,82. A Tabela 1 mostra a ordem de apresentação e o texto dos itens na versão traduzida para o português do Brasil e o valor do $\mathrm{CVC}_{\mathrm{i}}$ por item e para cada aspecto avaliado, em função 
dos quatro juízes especialistas. A versão final ressaltada pela Tabela 1 apresenta as alterações sugeridas pelo grupo de juízes.

A partir do total de itens, apenas 3 (15\%) deles tiveram algum tipo de problema relacionado ao $C V C_{i}$ : os itens 3, 16 e 20, mostrados na Tabela 1. Portanto, a tradução e a adaptação do QCA podem ser consideradas boas e suficientes para um instrumento adaptado à cultura brasileira. Em relação aos itens que tiveram deficiência no valor do $\mathrm{CVC}_{\mathrm{i}}$, as sugestões dos especialistas foram consi- deradas pelo painel multidisciplinar, realizando-se as alterações devidas, com o objetivo de dar forma final à Escala de Controle Atencional. 0 item 3, que apresentou problemas no $\mathrm{CVC}_{i}$ para a retrotradução, foi alterado para se adequar semanticamente à versão original. O termo "atento", que estava sendo usado inicialmente, foi mudado para o termo "concentrado", que melhorou a correspondência semântica com as versões em inglês. Após as sugestões, houve ajustes finais também nos itens 16 e 20. No item 16, a expressão "novas ideias

Tabela 1

Número da ordem de apresentação do item, o texto da versão final e o CVC para cada item calculado em função dos quatro juízes especialistas

\begin{tabular}{|c|c|c|c|c|}
\hline \multirow{2}{*}{ Número do Item } & \multirow{2}{*}{ Versão Final traduzida e adaptada para o português do Brasil } & \multicolumn{3}{|c|}{ CVC } \\
\hline & & Clareza & Coerência & Retrotradução \\
\hline 1 & $\begin{array}{l}\text { Tenho dificuldade de me concentrar em uma tarefa difícil quando há muito ba- } \\
\text { rulho em volta. }\end{array}$ & 0,9 & 1,0 & 0,8 \\
\hline 2 & $\begin{array}{l}\text { Quando preciso me concentrar para resolver um problema, tenho dificuldade de } \\
\text { focalizar minha atenção. }\end{array}$ & 0,8 & 0,9 & 0,9 \\
\hline 3 & $\begin{array}{l}\text { Mesmo quando estou concentrado trabalhando em algo, distraio-me com o que } \\
\text { está acontecendo em volta. }\end{array}$ & 1,0 & 1,0 & $0,6^{*}$ \\
\hline 4 & $\begin{array}{l}\text { Minha concentração é boa mesmo quando há música tocando em um ambiente } \\
\text { fechado. }\end{array}$ & 1,0 & 1,0 & 0,9 \\
\hline 5 & $\begin{array}{l}\text { Quando estou me concentrando, consigo focar minha atenção de forma que eu } \\
\text { não mais percebo o que está acontecendo ao redor em um ambiente fechado. }\end{array}$ & 0,8 & 0,8 & 0,8 \\
\hline 6 & $\begin{array}{l}\text { Quando estou lendo ou estudando, distraio-me facilmente quanto há pessoas } \\
\text { conversando em um ambiente fechado. }\end{array}$ & 0,8 & 1,0 & 0,8 \\
\hline 7 & $\begin{array}{l}\text { Quando estou tentando concentrar-me em algo, tenho dificuldade para bloque- } \\
\text { ar pensamentos que me distraem. }\end{array}$ & 0,8 & 0,8 & 0,9 \\
\hline 8 & Tenho dificuldade de me concentrar quando estou animado com algo. & 1,0 & 0,8 & 1,0 \\
\hline 9 & Quando estou concentrado, ignoro as sensações de fome e sede. & 1,0 & 0,9 & 1,0 \\
\hline 10 & Consigo mudar rapidamente de uma tarefa para outra. & 0,9 & 0,8 & 1,0 \\
\hline 11 & Levo certo tempo para realmente concentrar-me em uma nova tarefa. & 0,9 & 0,8 & 0,9 \\
\hline 12 & $\begin{array}{l}\text { Tenho dificuldade de coordenar minha atenção entre as tarefas de ouvir e escre- } \\
\text { ver quando tomo nota durante uma palestra ou aula. }\end{array}$ & 0,8 & 0,9 & 0,8 \\
\hline 13 & Consigo interessar-me rapidamente por outro assunto quando necessário. & 1,0 & 0,9 & 0,8 \\
\hline 14 & Para mim é fácil ler ou escrever ao mesmo tempo em que falo ao telefone. & 0,9 & 0,9 & 1,0 \\
\hline 15 & Tenho dificuldade em manter duas conversas ao mesmo tempo. & 1,0 & 0,9 & 0,9 \\
\hline 16 & Tenho dificuldade de ter novas ideias rapidamente. & 0,8 & $0,7^{*}$ & 0,9 \\
\hline 17 & $\begin{array}{l}\text { Após ser interrompido ou distraído, consigo facilmente retomar minha atenção } \\
\text { para o que estava fazendo. }\end{array}$ & 0,8 & 0,9 & 0,8 \\
\hline 18 & Quando fico distraído com um pensamento, é fácil desviar minha atenção dele. & 0,8 & 0,8 & 0,9 \\
\hline 19 & É fácil para mim alternar entre duas tarefas diferentes. & 1,0 & 1,0 & 0,9 \\
\hline 20 & $\begin{array}{l}\text { É difícil para mim trocar de uma forma de pensar sobre algo e olhá-lo por outro } \\
\text { ponto de vista. }\end{array}$ & 0,9 & $0,7^{*}$ & 1,0 \\
\hline
\end{tabular}

Nota: *Valores que apresentam CVC $<0,8$ sugerem que o item pode apresentar algum tipo de distorção ou problema no aspecto julgado. 
rápidas" foi substituída por "novas ideias rapidamente". No item 20, otermo "forma de pensamento" foi alterado para "forma de pensar".

\section{Discussão}

O objetivo do presente trabalho foi conduzir a tradução da ACS do inglês para o português do Brasil e adaptá-la semanticamente para o contexto brasileiro. Os resultados apresentaram evidências que sugerem que o QCA teve tradução e adaptação adequadas. Os três aspectos considerados para o julgamento da qualidade da escala - clareza global, coerência com o construto e correspondência semântica da retrotradução -, obtiveram $\mathrm{CVC}_{t}$ maiores que 0,8 . Isso sugere que o instrumento é coerente e bem adaptado (Hernández-Nieto, 2002).

Pormenorizando as dificuldades encontradas, os itens 4, 5 e 6 apresentaram a palavra "room", que sugere significados semanticamente diversos no português. Foi escolhido o termo "ambiente fechado", por representar um espaço físico generalizado, como uma sala de aula, um escritório ou um quarto. Qualquer dessas opções faz referência à palavra original, o que significa que todas deveriam ser abarcadas. A sugestão da palavra "cômodo" chegou a ser cogitada; contudo, o painel multidisciplinar considerou que "cômodo" estaria mais associado aos cômodos de uma casa. Nesse sentido, uma pessoa que estivesse preenchendo o questionário poderia imaginar que o item faria referência a um ambiente estritamente doméstico. Dado que o $C V C_{i}$ apresentou resultados satisfatórios para todos os aspectos julgados, a alteração pareceu adequada, sugerindo que o termo adaptou-se semanticamente.

O item 12 teve a inclusão dos termos "palestra ou aula" em sua tradução, pois o conceito de "lecture" engloba ambas as opções. A esse respeito, o painel multidisciplinar considerou importante a inclusão da palavra "aula", uma vez que muitas palestras não são acompanhadas de anotações pelos espectadores. Essa variável poderia influenciar as respostas de acordo com a experiência de cada respondente nos ambientes de palestra e confe- rência. Entretanto, a ideia de anotação na sala de aula, na opinião do painel multidisciplinar, deixa o item mais abrangente e faz com que cumpra seu propósito. Essa sugestão foi confirmada como uma boa proposta ao serem examinados os escores de $\mathrm{CVC}_{\mathrm{i}}$ para os três aspectos julgados, todos superiores a 0,8. Concluiu-se, portanto, que a adaptação foi coerente na opinião da equipe de juízes.

Segundo Hernández-Nieto (2002), valores de CVC menores que 0,8 sugerem que os juízes não estão de acordo em relação à adaptação de um item. Esse fenômeno ocorreu nos itens 3, 16 e 20 , porém em diferentes aspectos julgados. 0 item 3 teve resultado aquém do esperado no julgamento da retrotradução. Isso pode sugerir que esse item pode não ter conteúdo semântico equivalente ao original e apresentar carga fatorial em outra dimensão, não encontrada por Ólafsson et al. (2011). Para tanto, o termo "atento" foi substituído pelo termo "concentrado". No inglês a expressão original era "focused", que pode ser literalmente traduzida como "focado". No entanto, essa expressão pode ser pouco clara no contexto do item e sofrer de interpretações que levem a respostas que não estejam avaliando exatamente a atenção concentrada. O termo "atento" dá a sensação de atenção difusa ou dispersa, como se a pessoa estivesse atenta a diversos aspectos ambientais e suas possíveis alterações. A substituição sugerida pelos avaliadores foi "concentrado", o que facilita a compreensão semântica. O item quer saber se a pessoa mantém a atenção em uma tarefa ao longo do tempo, e essa pareceu a expressão mais adequada ao item.

O item 16 foi alterado após o cálculo do $\mathrm{CVC}_{\mathrm{i}}$ e das sugestões do grupo de especialistas. Dois especialistas sugeriram substituir a expressão "novas ideias rápidas" por "novas ideias rapidamente", o que foi acolhido pela equipe multidisciplinar. A primeira versão dá a noção de uma ideia que é caracterizada pela sua rapidez, não significando, necessariamente, que o modo como ela surge é rápido, mas somente que a ideia em si mesma caracteriza-se por ser rápida. Isso pode confundir o respondente ao considerar que o item trata de uma ideia apressada, ou mesmo impensada, o que não cons- 
titui a essência do construto avaliado. Após a introdução das sugestões na escala, fica claro que se trata de a ideia surgir rapidamente, independentemente de sua qualidade ou natureza. Isso fez com que o item se adaptasse melhor ao construto 'controle atencional' e ao contexto semântico brasileiro.

No item 20, a expressão "forma de pensamento" foi alterada para "forma de pensar", conforme sugestão de um dos especialistas. O resultado do $C V C_{i}$ do item para o aspecto da coerência não foi satisfatório, o que levou a equipe multidisciplinar a aceitar a sugestão e alterar o item. Na opinião do painel e do especialista, a expressão "forma de pensamento" está mais vinculada à noção de que um pensamento possui uma forma, o que se aproxima da noção de pensamento automático de Beck (1997). O pensamento automático consiste naquele que é construído previamente através das experiências do sujeito com o meio e que surge diante de novas experiências similares à primeira, na qual o pensamento foi construído. A expressão "forma de pensar" assemelha-se mais ao conceito de flexibilidade (Posner \& Rothbart, 1998), capacidade de alternar entre diferentes pensamentos, alterando a trajetória pela qual o sujeito chega à resposta a um estímulo.

As mudanças incorporadas à versão final do QCA parecem pertinentes e foram adotadas com o crivo e anuência dos especialistas, o que sugere que suas modificações foram decisivas no processo de adaptação cultural. A adaptação e tradução de um instrumento são processos trabalhosos e que exigem cuidado a fim de manter a correspondência com a escala original, como pôde ser demonstrado ao longo deste estudo. Isso permite comparações entre diferentes culturas e facilita pesquisas internacionais sobre o mesmo espectro de questões (Flaherty et al., 1988).

As escalas de autopreenchimento constituem ferramentas alternativas aos testes que avaliam o desempenho do sujeito em uma tarefa. Diversos são os instrumentos que fazem a mensuração da atenção através da performance, mas no Brasil ainda são escassas as medidas de preenchimento no tocante à atenção. O presente estudo é um marco inaugural na compreensão do QCA como um instrumento que completa essa lacuna. O uso de técnicas de tradução e adaptação de medidas de outros países é importante, pois permite comparações e estudos transculturais (International Test Commission, 2010). Essas técnicas devem seguir critérios rigorosos, como foi feito neste estudo, respeitando o contexto social e histórico do país de origem da escala e do país no qual a tradução será adaptada. Nesse sentido, a preocupação com os termos usados nos itens do QCA foi um dos alicerces deste trabalho.

Embora a presente pesquisa não tivesse como objetivo validar ou normatizar o QCA, sua contribuição não se limita a somente traduzir uma escala. Além de verificar a equivalência da versão traduzida do instrumento em relação à versão original, o derradeiro trabalho está em adequar e preparar a medida para o uso no contexto cultural brasileiro, respeitando as particularidades linguísticas e semânticas (Hambleton, 2005). Este primeiro trabalho abre as portas para pesquisadores trabalharem sobre as propriedades psicométricas do QCA, bem como para psicólogos clínicos usarem a escala como referência associada a medidas de ansiedade, e para os demais profissionais mensurarem a atenção através desse Questionário. As implicações da versão adaptada do QCA são as mais diversas, e o presente estudo buscou fornecer à literatura psicológica no Brasil uma ferramenta diferente na avaliação dos processos atencionais.

Apesar das boas características de tradução e adaptação semântica, o QCA ainda precisa passar por vários estudos psicométricos para se tornar uma nova opção de instrumento de medida para o controle atencional no Brasil. Isso significa que estudos usando as teorias clássicas dos testes, a análise da estrutura fatorial latente, a aplicação em amostras clínicas, bem como a análise das correlações do QCA com escalas que avaliam a depressão e ansiedade, como sugerido por Derryberry e Reed (2002) e por Ólafsson et al. (2011), ainda devem ser conduzidos em amostras no Brasil, para que a medida configure um instrumento suficientemente bom para ser usado pelos profissionais da área da saúde. 


\section{Referências}

Beck, J. (1997). Terapia cognitiva: teoria e prática. Porto Alegre: Artes Médicas.

Brickenkamp, R. (2000). Teste D2. São Paulo: Centro Editor de Testes e Pesquisa em Psicologia.

Broadbent, D. E., Cooper, P. F., FitzGerald, P., \& Parkes, K. R. (1982). The cognitive failures questionnaire (CFQ) and its correlates. British Journal of Clinical Psychology, 21(1), 1-16.

Cambraia, S. V. (2003). Atenção Concentrada - AC. São Paulo: Vetor.

Camilo, F. (2012). Teste de Atenção Seletiva - TAS. São Paulo: Vetor.

Carrasco, M. (2011). Visual attention: The past 25 years. Vision Research, 51(13), 1484-1525.

Conde, E. F. Q., Filgueiras, A., \& Lameira, A. P. (2009). Tempo de reação no futebol: a tarefa de compatibilidade estímulo-resposta como estratégia de treinamento. Coleção Pesquisa em Educação Física, 8(4), 11-18.

Conselho Federal de Psicologia. (2012). Sistema de avaliação dos testes psicológicos SATEPSI. Brasília: CFP. Recuperado em abril 14, 2012, de http://www.pol. org.br/satepsi/sistema/admin.cfm?lista1=sim

Cronbach, L. J. (1971). Essentials of psychological testing. New York: Harper \& Row Publishers.

Derryberry, D. (2002). Attention and voluntary self-control. Self and Identity, 1(2), 105-111.

Derryberry, D., \& Reed, M. A. (2002). Anxiety related attentional biases and their regulation by attentional control. Journal of Abnormal Psychology, 111, 225-236. http://dx.doi.org/10.1037/0021-843X.111.2.225

Duchesne, M., \& Mattos, P. (1997). Normatização de um teste computadorizado de atenção visual. Arquivos em Neuropsiquiatria, 55(1), 62-69.

Efstratopoulou, M., Janssen, R., \& Simons, J. (2012). Differentiating children with attention-deficit/ hyperactivity disorder, conduct disorder, learning disabilities and autistic spectrum disorders by means of their motor behavior characteristics. Research in Developmental Disabilities, 33(1), 196-204.

Figueiredo, V., Pinheiro, S., \& Nascimento, E. (1998). Teste de inteligência WISC-III: adaptando para a população brasileira. Psicologia Escolar e Educacional, 2(2), 101-107.

Filgueiras, A. (2011). A influência de características dos uniformes de times de futebol no tempo de reação manual em tarefas de orientação da atenção visual. Ciências \& Cognição, 16(2), 19-34.

Filgueiras, A. (2010). Abordagem neuropsicológica dos processos de orientação da atenção visuo-espacial e manutenção da concentração em atletas da categoria sub-13 de futebol de campo. Ciências \& Cognição,
Flaherty, J. A., Gavilia, M., Pathak, D., Mitchell, T., Wintrob, R., Richman, J. A., \& Birz, S. (1988). Developing instruments for cross-cultural psychiatric research. Journal of Nervous and Mental Disease, 176(5), 257-263.

Gazzaniga, M. S., \& Heatherton, T. F. (2007). Psychological Science: Mind, brain and behavior ( $2^{\text {nd }}$ ed.) New York: W. W. Norton \& Company.

Hambleton, R. K. (2005). Issues, designs, and technical guidelines for adapting tests into multiple languages and cultures. In R. K. Hambleton, P. F. Merenda, \& C. D. Spielberger (Eds.), Adapting educational and psychological tests for cross-cultural assessment (pp.3-38). Mahwah: Lawrence Erlbaum.

Hernández-Nieto, R. A. (2002). Contributions to statistical analysis. Mérida: Universidade de Los Andes.

International Test Commission. (2010). International Test Commission guidelines for translating and adapting tests. Received July 30, 2013, from http://www. intestcom.org/upload/sitefiles/40.pdf

Irwin, W., Davidson, R. J., Lowe, M. J., Mock, B. J., Sorenson, J. A., \& Turski, P. A. (1996). Human amygdala activation detected with echo-planar functional magnetic resonance imaging. NeuroReport, 7(11), 1765-1769.

Kiefer, M., Adams, S. C., \& Zovko, M. (2012). Attentional sensitization of unconscious visual processing: Topdown influences on masked priming. Advances in Cognitive Psychology, 8(1), 50-61.

Kimchi, R. (1982). Mental effort and task interference in auditory attention. Perception \& Psychophysics, 32(5): 473-480.

Kolb, B., \& Whishaw, I. Q. (2008). Fundamentals of human neuropsychology (6 $6^{\text {th }}$ ed.) New York: Worth Publishers.

Lonigan, C. J., \& Phillips, B. M. (2001). Temperamental influences on the development of anxiety disorders. In: M. W. Vasey \& M. R. Dadds (Orgs.), The developmental psychopathology of anxiety (pp.60-91). New York: Oxford University Press.

Nascimento, E. (2004). WAIS-III: Escala de Inteligência Wechsler para Adultos - manual para administração e avaliação. São Paulo: Casa do Psicólogo.

Norman, D. A., \& Shallice, T. (1986). Attention to action: Willed and automatic control of behaviour. In R. J. Davidson, G. E. Schwartz, \& D. Shapiro (Orgs.), Consciousness and self regulation (pp.1-18). NewYork: Plenum Press.

Ólafsson, R. P., Smári, J., Guðmundsdóttir, F., Ólafsdóttir, G., Harðardóttir, H. L., \& Einarsson, S. M. (2011). Self reported attentional control with the Attentional Control Scale: Factor structure and relationship with symptoms of anxiety and depression. Journal of Anxiety Disorders, 25(6), 777-782.

Posner, M. I. (1980). Orienting of Attention. The Quarterly Journal of Experimental Psychology, 32(1), 3-25. 
Posner, M. I., Snyder, R. R. C., \& Davidson, B. J. (1980). Attention and detection of signals. Journal of Experimental Psychology: General, 109(2), 160-174.

Posner, M. I., \& Rothbart, M. K. (1998). Attention, selfregulation and consciousness. Philosophical Transactions of the Royal Society of London B, 353(1377), 1915-1927.

Rueda, F. J. M. (2008). Teste de Atenção Concentrada (Teaco-FF). São Paulo: Casa do Psicólogo.

Rueda, F. J. M. (2009a). Teste de Atenção Dividida (TEADI). São Paulo: Casa do Psicólogo.

Rueda, F. J. M. (2009b). Teste de Atenção Alternada (TEALT). São Paulo: Casa do Psicólogo.

Schmidt, S. L., \& Manhães, A. C. (2001). Teste computadorizado da atenção visual (TCA Vis). Rio de Janeiro: Cognição.

Sisto, F. F., \& Castro, N. R. (2010). EASV: Escala de atenção seletiva visual. São Paulo: Casa do Psicólogo.
Sisto, F. F., Noronha, A. P. P., Bartholomeu, D., \& Rueda, F. J. M. (2006). Coleção AD e AS: Testes de Atenção Dividida e Sustentada. São Paulo: Vetor.

Small, D. M., Gitelman, D. R., Gregory, M. D., Nobre, A.C., Parrish, T. B., \& Mesulam, M. M. (2003). The posterior cingulate and medial prefrontal cortex mediate the anticipatory allocation of spatial attention. Neurolmage, 18(3), 633-641.

Spada, M. M., Georgiou, G. A., \& Wells, A. (2010). The relationship among metacognitions, attentional control, and state anxiety. Cognitive Behaviour Therapy, 39(1), 64-71.

Tonglet, E. C. (1999). Bateria de funções mentais para motorista. São Paulo: Vetor.

Tonglet, E. C. (2003). Bateria geral de funções mentais. São Paulo: Vetor.

Recebido: maio 20, 2013

Versão final: agosto 1, 2013

Aprovado: setembro 13, 2013 
